\title{
Le point sur la ventilation mécanique invasive - Importance des dispositifs médicaux dans la prévention des pneumonies acquises sous ventilation mécanique
}

\section{Latest developments for invasive mechanical ventilation - Importance of medical devices in preventing ventilator-associated pneumonia}

\author{
J.-D. Ricard · B. Bourreau Guérinière · M. Kerneis · S. Villard • D. Dreyfuss \\ (C) SRLF et Springer-Verlag France 2011
}

\section{Introduction}

La relation entre dispositifs médicaux (DM) et pneumonie nosocomiale n'est pas nouvelle, mais s'est diamétralement modifiée ces dernières années. On est passé d'un lien de causalité à un « lien de prévention ». Alors qu'à la fin des années 1960, plusieurs épidémies de pneumonies nosocomiales ont été rapportées à une contamination des dispositifs d'aérosol et d'humidification [1,2] ; en 2010, c'est — par exemple — l'utilisation d'un nouveau modèle de sonde d'intubation munie d'un orifice permettant le drainage des sécrétions s'accumulant dans l'espace sous-glottique qui est associée à une réduction significative des pneumonies acquises sous ventilation mécanique (PAVM) [3]. Ce chapitre a pour but d'analyser et de faire la synthèse des données disponibles sur l'impact des DM sur la survenue des PAVM.

\section{Données historiques}

\section{Nébuliseurs et humidificateurs}

Comme indiqué plus haut, la responsabilité dans la survenue de pneumonies nosocomiales de DM type générateurs d'aérosol et humidificateurs a été reconnue dans le milieu des années 1960 [1,2]. Plusieurs épidémies de pneumonies à bacilles à Gram négatif ont été rapportées, et la contamina-

\footnotetext{
J.-D. Ricard $(\varangle) \cdot$ B. Bourreau Guérinière $\cdot$ M. Kerneis

S. Villard · D. Dreyfuss

Assistance Publique - Hôpitaux de Paris,

Hôpital Louis Mourier,

Service de Réanimation Médico-Chirurgicale,

F-92700, Colombes, France

e-mail : jean-damien.ricard@lmr.aphp.fr
}

Unité Inserm U722, UFR de médecine, université Paris-Diderot, Paris-VII, PRES Sorbonne Paris Cité, Paris, France tion des nébuliseurs a été très vraisemblablement la cause de ces épidémies. Ainsi, en 1967, Mertz et al. ont décrit cinq cas mortels de pneumonie nécrosante due à Klebsiella pneumoniae en l'espace d'un mois, chez des patients ventilés mécaniquement ayant reçu des bronchodilatateurs nébulisés [1]. Vers la même époque, Grieble et al. ont rapporté une épidémie de pneumonie à Pseudomonas aeruginosa liée à la contamination d'un humidificateur non chauffant [2]. Les nébuliseurs contaminés produisaient de très fines particules, vecteur idéal pour permettre aux bactéries d'atteindre le parenchyme pulmonaire. Ces équipements ne sont plus utilisés pour humidifier les gaz inspirés des patients ventilés comme par le passé, mais le risque demeure comme récemment illustré par la survenue de plusieurs épidémies à Ralstonia mannitolilytica en pédiatrie avec des systèmes d'oxygénothérapie fonctionnant avec une base chauffante [4].

\section{Circuits des ventilateurs}

En gardant le même raisonnement, les circuits des ventilateurs - lorsqu'ils sont utilisés avec un humidificateur chauffant - ont été considérés comme des facteurs de risque de PAVM [5,6] en raison de leur contamination rapide et importante par des bactéries pathogènes [5,7-9]. Ainsi, Grieble et al. ont étudié la colonisation des circuits et des ventilateurs au cours des 24 premières heures suivant le changement du circuit. Ils ont trouvé une colonisation rapide : $33 \%$ des circuits étaient colonisés deux heures seulement après leur changement, $64 \%$ à 12 heures et $80 \%$ à 24 heures. Le niveau médian de la colonisation à 24 heures était de $7 \times 10^{4} \mathrm{ufc} / \mathrm{ml}$ [5]. Les bactéries isolées de la condensation des circuits étaient celles trouvées dans les sécrétions trachéobronchiques des patients $[5,7,10]$. Les DM les plus proches du patient sont ceux les plus lourdement et les plus rapidement contaminés : dans l'étude de Craven et al. [5], plus de $90 \%$ des adaptateurs pivotants (raccord annelé) étaient contaminés alors que ce chiffre n'était que de $33 \%$ 
pour la partie plus distale du circuit. Le taux de formation de la condensation dans le circuit était rapide $(30 \mathrm{ml} / \mathrm{h})$ et le niveau de la colonisation dans le condensat était élevé (niveau médian : $2 \times 10^{5} \mathrm{ufc} / \mathrm{ml}$ ). De tels niveaux de micro-organismes représentent évidemment un danger important de contamination croisée lors de la vidange de ces condensats, mais ont également été considérés comme un facteur de risque de PAVM [5]. Dans une autre étude, Craven et al. ont étudié les facteurs de risque de VAP chez plus de 200 patients nécessitant la ventilation mécanique et constaté que les changements des circuits des ventilateurs toutes les 24 heures plutôt que toutes les 48 heures faisaient partie de ces risques [6]. Ce résultat a sérieusement mis en doute la pertinence d'un changement des circuits des ventilateurs toutes les 24 heures comme cela était couramment fait à l'époque. Lareau et al. ont été les premiers à montrer que l'augmentation de la durée d'utilisation de circuits des ventilateurs de 8 à 24 heures n'était pas associée à une augmentation de l'incidence de VAP ou du niveau de contamination du circuit [11]. Ce résultat original a été confirmé par Craven et al. qui ont montré un même niveau de la colonisation circuit que ceux-ci étaient changés toutes les $24 \mathrm{ou}$ 48 heures [9]. Il a ensuite été démontré qu'en fait, les circuits des ventilateurs n'avaient pas besoin d'être changés pendant toute la période de ventilation mécanique d'un patient donné [12]. Dans cette étude randomisée, les circuits des ventilateurs étaient soit changés toutes les 48 heures, soit pas du tout. Une étude microbiologique exhaustive a montré des taux similaires de contamination des circuits, et surtout, les mêmes taux de PAVM entre les deux groupes [12]. Ces résultats pionniers ont été confirmés par la suite par plusieurs équipes [13-16], conduisant à une évolution radicale des recommandations du CDC pour la prévention des PAVM, passant d'une recommandation pour un changement quotidien des circuits [17] à aucun changement de routine, sauf en cas de souillure visible ou de défection ou détérioration physique du circuit [18]. Évidemment, une telle modification des pratiques se traduit par des économies considérables [12].

\section{Prévention de la contamination des circuits des ventilateurs}

Ainsi qu'on l'a vu plus haut, les circuits des ventilateurs sont rapidement et fortement contaminés lorsqu'ils sont utilisés avec un humidificateur chauffant [5,7-9]. À cet égard, l'utilisation des échangeurs de chaleur et d'humidité (ECH) confère un avantage majeur à celle des humidificateurs chauffants. En effet, plusieurs études ont montré de façon reproductible la réduction de la contamination des circuits des ventilateurs avec un ECH par rapport aux humidificateurs chauffants. Martin et al. ont trouvé que $11 \%$ des circuits utilisés avec un ECH étaient contaminés contre $54 \%$ avec un humidificateur chauffant $(p<0,01)[19]$. Lorsque les patients avaient un prélèvement bronchique positif, le même agent pathogène était retrouvé sur la pièce en $\mathrm{Y}$, dans $10 \%$ des cas avec l'ECH et dans $65 \%$ avec un humidificateur chauffant $(p<0,001)$. Des résultats très similaires et concordants ont été trouvés par d'autres [20-22]. Comme indiqué ci-dessus, les bactéries présentes dans les circuits proviennent de la flore respiratoire des patients, expliquant pourquoi dans ces études, colonisations oropharyngée et trachéale n'étaient pas différentes, que les patients soient ventilés avec un ECH ou avec un humidificateur chauffant [22].

\section{Influence du dispositif d'humidification}

S'il y a pu avoir débat pour savoir si un dispositif d'humidification plus qu'un autre favorisait ou au contraire protégeait des PAVM, ce débat n'a plus lieu d'être $[23,24]$. En effet, comme indiqué ci-dessus, les bactéries qui sont retrouvées dans les circuits proviennent de la flore respiratoire des patients. Dès lors, on imagine mal comment un dispositif d'humidification - quel qu'il soit - puisse influencer le développement d'une PAVM. Cette constatation a été faite très tôt par Dreyfuss et al., dans une étude comparant l'incidence des PAVM entre deux groupes de patients, l'un ventilé avec un ECH et l'autre avec un humidificateur chauffant [22]. Même si la contamination bactérienne des circuits était plus importante lorsque les patients étaient ventilés avec un humidificateur chauffant ; cela n'avait aucune incidence sur le taux de PAVM. Ce résultat a été confirmé par la suite par plusieurs équipes.

\section{Influence des sondes d'intubation}

Une des voies par lesquelles des bactéries pathogènes peuvent atteindre le parenchyme pulmonaire et entraîner une pneumonie est la micro-inhalation occulte de sécrétions contaminées accumulées dans l'espace sous-glottique au-dessus du ballonnet de la sonde d'intubation. À l'occasion d'une perte d'étanchéité du ballonnet, ces sécrétions peuvent descendre le long de l'arbre trachéobronchique et contaminer le poumon. Plusieurs arguments - indirects — plaident en faveur de ce mécanisme. Parmi eux, la détection - dans les sécrétions trachéobronchiques - de particules radiomarquées par méthode isotopique instillée dans l'estomac par une sonde gastrique chez des patients intubés et ventilés atteste d'un passage de ces particules depuis l'oropharynx vers le poumon, au travers de la sonde d'intubation et plus particulièrement de son ballonnet [25]. Cette détection signifie qu'à un moment donné, l'étanchéité de la trachée n'a pas été parfaite. La fonction du ballonnet de la sonde d'intubation est justement d'assurer cette étanchéité, celle-ci étant obtenue grâce à un gonflement adéquat du ballonnet. Cette adéquation fait intervenir le volume du ballonnet et sa 
pression, et comme on le verra plus tard, sa forme et le matériau qui le compose. Concernant les PAVM, une étude s'intéressant à ses facteurs de risque avait trouvé qu'une pression du ballonnet inférieur à $20 \mathrm{cmH}_{2} \mathrm{O}$ constituait un facteur de risque de PAVM [26]. Cette étude a été à la base de la recommandation, jamais réévaluée, selon laquelle la pression minimale à laquelle devait être gonflé un ballonnet au cours de la ventilation mécanique était de $20 \mathrm{cmH}_{2} \mathrm{O}$.

Les difficultés majeures auxquelles sont confrontées les équipes infirmières résident dans la détermination de cette pression, la fréquence de sa surveillance et la meilleure façon de la réguler. En effet, hormis la recommandation de maintenir une pression du ballonnet supérieure à 20-25 $\mathrm{cmH}_{2} \mathrm{O}$, il n'existe aucune recommandation concernant la meilleure méthode pour déterminer cette pression (manomètre manuel, dispositif électronique ?), la fréquence optimale de cette surveillance et la technique appropriée pour réguler cette pression. Quelques données dans la littérature récente permettent d'apporter des éléments de réponse à ces différentes questions. Une surveillance " occasionnelle » de la pression du ballonnet avec un manomètre manuel ne permet pas de respecter les objectifs de pression d'une proportion suffisante de patients. Ainsi, dans une étude comparant surveillance avec un manomètre manuel et surveillance automatisée, une valeur inférieure à $20 \mathrm{cmH}_{2} \mathrm{O}$ était rapportée dans $45 \%$ des patients du groupe « surveillance manuelle » contre seulement $0,7 \%$ du groupe « automatique » [27]. Cependant, il est important de noter que cette différence considérable ne s'est pas traduite en une plus grande incidence de PAVM. Plusieurs raisons peuvent être avancées pour expliquer ce résultat décevant : un mauvais contrôle des autres facteurs de risques de PAVM, une puissance statistique insuffisante, l'utilisation d'une sonde d'intubation « suboptimale » (absence de canal permettant l'aspiration sous-glottique, ni ballonnet en polyuréthane [PU]) et la fabrication artisanale du régulateur automatique de la pression ; avec peut-être des temps et des amplitudes de réaction inadaptés. L'importance de ce dernier point a été récemment illustrée par Weiss et al. [28]. Dans cette étude, les auteurs ont comparé plusieurs régulateurs de pression sur banc d'essai et ont noté une correction trop rapide des régulateurs face à une augmentation de la pression, entraînant une moindre étanchéité des ballonnets. Il faut espérer qu'une simple modification de l'algorithme de correction de la pression peut corriger ce défaut. En tout état de cause, il semble souhaitable de recommander l'automatisation de la mesure de la pression, la meilleure façon de la réguler restant à préciser.

\section{Matériau du ballonnet de la sonde d'intubation}

Après l'avènement du polychlorure de vinyle (PVC) dans de nombreux DM, ses inconvénients dans les ballonnets des sondes d'intubation ont été récemment mis en exergue dans plusieurs études. La raison tient au fonctionnement de ces ballonnets dit HVLP pour high volume, low pressure. En effet, ce n'est pas tant la pression du ballonnet, mais le volume qu'il occupe dans la trachée qui assure l'étanchéité de celle-ci. Or, le volume maximal d'un ballonnet HVLP est bien supérieur à celui - à un étage donné - de la trachée. Il en résulte donc que le dépliement du ballonnet (obtenu par son gonflage) n'est pas complet, et d'authentiques canaux se forment du fait de repliements de la paroi du ballonnet, contraint par la paroi trachéale. Ce phénomène est d'autant plus important que la paroi du ballonnet est épaisse. Une étude scannographique a documenté la formation de ces canaux de façon très convaincante [29]. Cette étude a également montré qu'il n'y avait presque plus de replis lorsque les ballonnets étaient fabriqués à partir de $\mathrm{PU}, \mathrm{du}$ fait d'une paroi du ballonnet beaucoup plus fine. Il semblerait que cette finesse n'entraine pas une plus grande fragilité du ballonnet, bien au contraire, le PU étant plus résistant que le PVC. Des données in vitro ont par la suite mis en évidence une bien meilleure étanchéité des ballonnets en PU [30].

In vivo, Poelaert et al. ont montré une réduction des pneumonies postopératoires précoces après chirurgie cardiaque chez les patients ventilés en peropératoire avec une sonde munie d'un ballonnet en PU par rapport avec un ballonnet en PVC [31]. En faisant appel à un dosage original de pepsine, Nseir et al. ont mesuré des concentrations beaucoup plus faibles de cette endoprotéase digestive du suc gastrique dans les sécrétions trachéales des patients intubés avec une sonde à ballonnet en PU par rapport à ceux ayant une sonde à ballonnet en PVC ; confirmant le concept de microinhalation autour et « au travers » du ballonnet comme possible voie de passage des bactéries au cours des PAVM [32].

Parallèlement au matériau, la forme du ballonnet peut également influencer son efficacité, et un ballonnet en forme de poire ou conique pourrait conceptuellement offrir de meilleures performances, même s'il n'y a pour le moment aucune donnée en ce sens. Dans le travail de Nseir et al. cité ci-dessus, il n'y avait pas de différence dans les concentrations de pepsine entre les sondes à ballonnet en PU de forme cylindrique et ceux en PU, mais de forme conique [32]. Pour compliquer le choix des équipes, les fabricants proposent différentes combinaisons : la sonde classique (actuellement la plus répandue en France) avec un ballonnet en PVC de forme « standard» (cylindrique) ; ballonnet en PU de forme standard, ballonnet en PVC avec la nouvelle forme dite conique ou en poire ; ballonnet en PU avec la nouvelle forme. Il n'existe actuellement aucune donnée permettant d'établir l'intérêt et la contribution respectifs de ces différents éléments dans la prévention des PAVM. Une étude est en cours pour justement déterminer si l'une des combinaisons confère un avantage sur les autres en matière d'étanchéité. Un défaut de cette dernière sera défini par 
l'identification - dans les sécrétions trachéobronchiques de bactéries précédemment isolées dans l'oropharynx des patients. Cette étude, appelée TOPCUFF, conduite par Benoit Misset, et à laquelle nous participons, devrait permettre d'apporter des réponses à même d'aider les équipes dans le choix de la sonde d'intubation.

Dans le même ordre d'esprit, une autre option — séduisante d'un point de vue physiopathologique - est la possibilité d'aspirer les sécrétions sous-glottiques qui se sont accumulées au-dessus du ballonnet. L'intérêt potentiel de cette technique est d'éviter - en aspirant régulièrement l'espace sous-glottique - que ces sécrétions ne soient inhalées du fait d'une baisse de l'étanchéité du ballonnet, ou de leur descente au travers des chenaux formés par les replis de la paroi du ballonnet évoqués plus haut. Mahul et al. furent les premiers à montrer une réduction des PAVM chez les patients intubés avec une sonde permettant l'aspiration des sécrétions sous-glottiques [33]. Par la suite, d'autres études ont confirmé l'intérêt potentiel de ces sondes, mais leur bénéfice semble résider surtout dans la réduction des pneumonies précoces [34]. Cela explique sans doute le fait qu'elles ne soient pas beaucoup utilisées. Cette impression est corroborée par les résultats d'une enquête européenne récente [35] réalisée auprès de plus 1500 médecins et infirmières de réanimation, montrant que seuls $30 \%$ des répondeurs déclaraient utiliser ces sondes.

Ces chiffres se modifieront peut-être dans un avenir proche après la publication de deux études randomisées contrôlées montrant une réduction nette des PAVM grâce à ces sondes $[3,36]$. Fait important, cette réduction porte non seulement sur les PAVM précoces, mais aussi et surtout, sur les PAVM tardives. Il faut cependant remarquer que la sonde de l'étude de Lorente et al. comportait non seulement un port latéral pour aspirer les sécrétions sous-glottiques, mais était également munie d'un ballonnet en PU. Ainsi, la part respective de chacun de ces éléments dans la réduction des PAVM ne peut être déterminée [36]. Un dernier point important pour les équipes infirmières concernant ces sondes mérite discussion. Il existe en effet plusieurs façons de réaliser l'aspiration sous-glottique, soit de façon automatique, avec un appareil dédié et l'aspiration peut être alors soit continue, soit intermittente ; soit de façon manuelle, par l'infirmière à intervalle régulier. Il n'existe aucune donnée dans la littérature permettant d'affirmer qu'une technique est meilleure que l'autre. Une étude expérimentale a montré le risque potentiel de lésions trachéales avec une aspiration automatique continue [37], mais ces lésions n'ont jamais été rapportées chez l'homme. Le seul argument - à notre avis — pour préférer une technique plutôt qu'une autre est le fait que les deux études les plus récentes et les plus convaincantes en termes de réduction des PAVM ont utilisé la même technique : aspiration intermittente manuelle, réalisée une fois par heure avec une seringue de $10 \mathrm{ml}[3,36]$.

\section{Sonde imprégnée en argent}

Tout DM quel qu'il soit est susceptible d'être recouvert d'un biofilm, dès lors qu'il se trouve en contact avec des micro-organismes. La sonde d'intubation n'échappe pas à la règle, et ce phénomène a été illustré grâce à la microscopie électronique [38]. La responsabilité de ce biofilm comme réservoir de bactéries susceptibles d'entraîner des PAVM a été également montrée [39]. Plusieurs études expérimentales ont donc cherché à évaluer l'intérêt d'une sonde d'intubation imprégnée de nitrate d'argent afin de limiter la formation de biofilm. Une étude clinique de grande envergure, randomisée contrôlée a comparé l'incidence des PAVM selon que les patients étaient intubés avec une sonde « standard» ou avec une sonde imprégnée en argent. Les résultats de cette étude sont décevants. Certes, les auteurs rapportent une diminution de l'incidence des PAVM dans le groupe sonde imprégnée d'argent, mais cela n'a aucun impact sur le devenir des patients ni en termes de mortalité ni de durée de séjour. De plus, l'éditorial qui accompagnait l'article soulignait d'une part, le fait qu'il ne suffisait que de trois PAVM supplémentaires dans le groupe « sonde imprégnée en argent» pour que la différence ne soit plus significative et d'autre part, le fait qu'il y avait plus de patients BPCO dans le groupe témoin [40]. Le coût prohibitif de la sonde (de l'ordre de 90 \$) lié à une efficacité discutable ne laisse actuellement aucune place pour ce type de DM dans notre stratégie de prévention des PAVM.

\section{Système clos d'aspiration trachéale}

Il est reconnu depuis très longtemps que l'aspiration trachéale classique dite ouverte (ouverture du circuit, introduction d'un cathéter stérile, aspiration, puis retrait du cathéter) s'accompagne d'une dispersion plus ou moins importante de gouttelettes. Lorsque le patient est porteur de bactéries dans ses sécrétions trachéales, cette dispersion est responsable d'une contamination bactérienne de l'environnement, et les bactéries peuvent être retrouvées à près d'un mètre de la sonde d'intubation [41]. Cette contamination est considérablement réduite lorsque l'on utilise un système clos [41] composé d'un cathéter protégé par une gaine et relié à la sonde d'intubation par un système de valve qui permet, sans ouvrir le circuit du respirateur, d'introduire le cathéter dans la sonde d'intubation et d'aspirer le patient. Plusieurs études ont évalué l'intérêt de ce système dans le but de réduire l'incidence des PAVM. Faisant le point de toutes ces études, deux méta-analyses ont montré l'absence d'impact du système clos par rapport au système ouvert sur le taux de PAVM $[42,43]$. Ces dispositifs doivent-ils être pour autant abandonnés ? Trois autres aspects cruciaux ne sont jamais abordés dans les études cliniques et ne sont pas 
pris en compte dans ces méta-analyses. Le premier concerne la réduction majeure du risque de dérecrutement et de désaturation au cours de l'aspiration trachéale permise par les systèmes clos $[44,45]$, le deuxième la réduction considérable de la contamination de l'extérieur de la sonde d'intubation et des mains du personnel soignant engendrée par un système clos [46]. Le troisième est l'avantage majeur des systèmes clos en matière de protection du personnel soignant, soit vis-à-vis d'agents pathogènes aérocontaminants (tuberculose, SRAS ou virus de la grippe H1N1) soit vis-à-vis $\mathrm{du}$ risque ophtalmologique, d'authentiques kératites (à $P$. aeruginosa par exemple) ayant été décrites chez des soignants, avec perte ou réduction de la vision [47].

$\mathrm{Au}$ terme de cette revue de la littérature, une synthèse forcément sujette à caution — du choix des DM à utiliser, dans l'optique de la prévention des PAVM. Elle devra être adaptée aux habitudes locales, et surtout, pourra évoluer en fonction de nouvelles données de la littérature :

- humidification.

Choix : filtre échangeur de chaleur et d'humidification

Justification :

- aucun impact sur les PAVM ;

- mais prévention de la contamination des circuits des respirateurs ;

- réduction du risque de transmission croisée ;

- réduction des coûts, et simplicité d'utilisation ;

- aspiration trachéale.

Système clos (pour un certain nombre de patients : hypoxémie sévère, niveaux de pression expiratoire positive élevés, sécrétions bronchiques très abondantes, colonisation trachéobronchique par des bactéries multirésistantes, infection respiratoire par des pathogènes aéroportés).

Justification :

- aucun impact sur les PAVM ;

- mais réduction du risque de transmission croisée ;

- protection du personnel soignant +++ ;

- réduction des coûts (au-delà de $48 \mathrm{~h}$ de ventilation mécanique);

- prévention du dérecrutement et de l'hypoxémie induits par l'aspiration trachéale.

Pour les autres, système ouvert ;

- sonde d'intubation :

- aspiration sous-glottique : oui.

Justification :

- impact sur les PAVM précoces et tardives ;

- imprégnation en argent : non.

Justification :

o impact limité sur les PAVM ;

○ coût prohibitif ;

- ballonnet de la sonde :

- forme : attente des résultats de l'étude TOPCUFF ;

- matériau : attente des résultats de l'étude TOPCUFF.

\section{Conclusion}

Après avoir été parfois à l'origine même d'épidémie de pneumonies nosocomiales, les DM proposent maintenant des caractéristiques et des fonctionnalités leur permettant au contraire - de participer à la prévention des pneumonies nosocomiales. Pour certains de ces DM, la preuve de l'efficacité de leur fonctionnalité est faite, alors que pour d'autres, des études sont en cours. Il est important dans tous les cas de confronter le bénéfice réel obtenu en termes de réduction des PAVM au coût de ces DM.

Enfin, il ne faut pas perdre de vue que l'utilisation de ces DM ne dispensera jamais les équipes - bien au contraire d'une application stricte et rigoureuse de l'ensemble des mesures universelles de prévention des infections nosocomiales, au premier rang desquelles se trouve l'hygiène des mains.

Conflit d'intérêt : Jean-Damien Ricard participe à un groupe de travail européen sur la prévention des PAVM, bénéficiant d'un soutien financier par la société Covidien.

\section{Références}

1. Mertz JJ, Scharer L, McClement JH (1967) A hospital outbreak of Klebsiella pneumoniae from inhalation therapy with contaminated aerosol solutions. Am Rev Respir Dis 95(3):454-60

2. Grieble HG, Colton FR, Bird TJ, et al (1970) Fine-particle humidifiers. Source of Pseudomonas aeruginosa infections in a respiratory-disease unit. N Engl J Med 282(10):531-5

3. Lacherade JC, De Jonghe B, Guezennec P, et al (2010) Intermittent subglottic secretion drainage and ventilator-associated pneumonia: a multicenter trial. Am J Respir Crit Care Med 182:910-7

4. Jhung MA, Sunenshine RH, Noble-Wang J, et al (2007) A national outbreak of Ralstonia mannitolilytica associated with use of a contaminated oxygen-delivery device among pediatric patients. Pediatrics 119(6):1061-8

5. Craven DE, Goularte TA, Make BJ (1984) Contaminated condensate in mechanical ventilator circuits. A risk factor for nosocomial pneumonia. Am Rev Respir Dis 129:625-8

6. Craven DE, LM Kunches, Kilinsky V, et al (1986) Risk factors for pneumonia and fatality in patients receiving continuous mechanical ventilation. Am Rev Respir Dis 133(5):792-6

7. Comhaire A, Lamy M (1981) Contamination rate of sterilized ventilators in an ICU. Crit Care Med 9(7):546-8

8. Christopher KL, Saravolatz LD, Bush TL, Conway WA (1983) The potential role of respiratory therapy equipment in cross infection. A study using a canine model for pneumonia. Am Rev Respir Dis 128(2):271-5

9. Craven DE, Connolly MG Jr, Lichtenberg DA, et al (1982) Contamination of mechanical ventilators with tubing changes every 24 or 48 hours. N Engl J Med 306(25):1505-9

10. Ricard JD, Hidri N, Blivet A, et al (2003) New heated breathing circuits do not prevent condensation and contamination of ventilator circuits with heated humidifiers. [abstract]. Am J Respir Crit Care Med 167:A861

11. Lareau SC, Ryan KJ, Diener CF (1978) The relationship between frequency of ventilator circuit changes and infectious hazard. Am Rev Respir Dis 118(3):493-6 
12. Dreyfuss D, Djedaini K, Weber P, et al (1991) Prospective study of nosocomial pneumonia and of patient and circuit colonization during mechanical ventilation with circuit changes every 48 hours versus no change. Am Rev Respir Dis 143(4 Pt 1):738-43

13. Hess D, Burns E, Romagnoli D, Kacmarek RM (1995) Weekly ventilator circuit changes. A strategy to reduce costs without affecting pneumonia rates. Anesthesiology 82(4):903-11

14. Kollef MH, Ward S (1998) The influence of mini-BAL cultures on patient outcomes: implications for the antibiotic management of ventilator-associated pneumonia. Chest 113(2):412-20

15. Kotilainen HR, Keroack MA (1997) Cost analysis and clinical impact of weekly ventilator circuit changes in patients in intensive care unit. Am J Infect Control 25(2):117-20

16. Long MN, Wickstrom G, Grimes A, et al (1996) Prospective, randomized study of ventilator-associated pneumonia in patients with one versus three ventilator circuit changes per week. Infect Control Hosp Epidemiol 17(1):14-9

17. Simmons BP, Wong ES (1982) Guideline for prevention of nosocomial pneumonia. Infect Control 3(4):327-33

18. Tablan OC, Anderson LJ, Besser R, et al (2004) Guidelines for preventing health-care-associated pneumonia, 2003: recommendations of $\mathrm{CDC}$ and the Healthcare Infection Control Practices Advisory Committee. MMWR Recomm Rep 53(RR-3):1-36

19. Martin C, Perrin G, Gevaudan MJ, et al (1990) Heat and moisture exchangers and vaporizing humidifiers in the intensive care unit. Chest 97(1):144-9

20. Branson RD, Davis KJ, Campbell RS, et al (1993) Humidification in the intensive care unit. Prospective study of a new protocol utilizing heated and humidification and a hygroscopic condenser humidifier. Chest 104:1800-5

21. Boots RJ, Howe S, George N, et al (1997) Clinical utility of hygroscopic heat and moisture exchangers in intensive care patients. Crit Care Med 25(10):1707-12

22. Dreyfuss D, Djedaïni K, Gros I, et al (1995) Mechanical ventilation with heated humidifiers or heat and moisture exchangers: effects on patient colonization and incidence of nosocomial pneumonia. Am J Respir Crit Care Med 151(4):986-92

23. Ricard JD (2007) Gold standard for humidification: heat and moisture exchangers, heated humidifiers, or both? Crit Care Med 35(12):2875-6

24. Ricard JD, Boyer A, Dreyfuss D (2006) The effect of humidification on the incidence of ventilator-associated pneumonia. Respir Care Clin N Am 12(2):263-73

25. Torres A, Serra-Batlles J, Ros E, et al (1992) Pulmonary aspiration of gastric contents in patients receiving mechanical ventilation: the effect of body position. Ann Intern Med 116(7):540-3

26. Rello J, Sonora R, Jubert P, et al (1996) Pneumonia in intubated patients: role of respiratory airway care. Am J Respir Crit Care Med 154(1):111-5

27. Valencia M, Ferrer M, Farre R, et al (2007) Automatic control of tracheal tube cuff pressure in ventilated patients in semirecumbent position: a randomized trial. Crit Care Med 35(6):1543-9

28. Weiss M, Doell C, Koepfer N, et al (2009) Rapid pressure compensation by automated cuff pressure controllers worsens sealing in tracheal tubes. Br J Anaesth 102(2):273-8. Epub 2008 Dec 25

29. Dullenkopf A, Gerber A, Weiss M (2003) Fluid leakage past tracheal tube cuffs: evaluation of the new Microcuff endotracheal tube. Intensive Care Med 29(10):1849-53. Epub 2003 Aug 16

30. Lucangelo U, Zin WA, Antonaglia V, et al (2008) Effect of positive expiratory pressure and type of tracheal cuff on the incidence of aspiration in mechanically ventilated patients in an intensive care unit. Crit Care Med 36(2):409-13

31. Poelaert J, Depuydt P, De Wolf A, et al (2008) Polyurethane cuffed endotracheal tubes to prevent early postoperative pneumonia after cardiac surgery: a pilot study. J Thorac Cardiovasc Surg 135(4):771-6

32. Nseir S, Zerimech F, De Jonckheere J, et al (2010) Impact of polyurethane on variations in tracheal cuff pressure in critically ill patients: a prospective observational study. Intensive Care Med 36(7):1156-63

33. Mahul P, Auboyer C, Jospe R, et al (1992) Prevention of nosocomial pneumonia in intubated patients: respective role of mechanical subglottic secretions drainage and stress ulcer prophylaxis. Intensive Care Med 18(1):20-5

34. Dezfulian C, Shojania K, Collard HR, et al (2005) Subglottic secretion drainage for preventing ventilator-associated pneumonia: a meta-analysis. Am J Med 118(1):11-8

35. Ricard JD, Conti G, Boucherie M, et al (2008) Vapaway project: a european audit of vap prevention practices. Intensive Care Med 34(suppl1):S36

36. Lorente L, Lecuona M, Jimenez A, et al (2007) Influence of an endotracheal tube with polyurethane cuff and subglottic secretion drainage on pneumonia. Am J Respir Crit Care Med 176 (11):1079-83. Epub 2007 Sep 13

37. Berra L, De Marchi L, Panigada M, et al (2004) Evaluation of continuous aspiration of subglottic secretion in an in vivo study. Crit Care Med 32(10):2071-8

38. Feldman C, Kassel M, Cantrell J, et al (1999) The presence and sequence of endotracheal tube colonization in patients undergoing mechanical ventilation. Eur Respir J 13(3):546-51

39. Adair CG, Gorman SP, Feron BM, et al (1999) Implications of endotracheal tube biofilm for ventilator-associated pneumonia. Intensive Care Med 25(10):1072-6

40. Chastre J (2008) Preventing ventilator-associated pneumonia: could silver-coated endotracheal tubes be the answer? JAMA 300(7):842-4

41. Cobley M, Atkins M, Jones PL (1991) Environmental contamination during tracheal suction. A comparison of disposable conventional catheters with a multiple-use closed system device. Anaesthesia 46(11):957-61

42. Vonberg RP, Eckmanns T, Welte T, Gastmeier P (2006) Impact of the suctioning system (open vs closed) on the incidence of ventilation-associated pneumonia: meta-analysis of randomized controlled trials. Intensive Care Med 32(9):1329-35

43. Jongerden IP, Rovers MM, Grypdonck MH, Bonten MJ (2007) Open and closed endotracheal suction systems in mechanically ventilated intensive care patients: a meta-analysis. Crit Care Med 35(1):260-70

44. Cereda M, Villa F, Colombo E, et al (2001) Closed system endotracheal suctioning maintains lung volume during volume-controlled mechanical ventilation. Intensive Care Med 27(4):648-54

45. Maggiore SM, Lellouche F, Pigeot J, et al (2003) Prevention of endotracheal suctioning-induced alveolar derecruitment in acute lung injury. Am J Respir Crit Care Med 167(9):1215-24

46. Ricard JD, Eveillard M, Martin Y, et al (2011) Closed system tracheal syctioning reduces health care workers hand and equipment contamination. Am J Infect Control (in press)

47. Ezra DG, Goyal S, Moosavi R, et al (2004) Microbial keratitis in ITU staff: an occupational hazard? Anaesthesia 59(12):1221-3 